\title{
Unitarity violation in non-abelian Pauli-Villars regularization
}

\author{
J.H. León ${ }^{\text {a }}$, C.P. Martin ${ }^{b}$, F. Ruiz Ruiz ${ }^{\mathrm{c}}$ \\ a Departamento de Física Teórica, C-XI, Universidad Autónoma de Madrid, Cantoblanco, 28049 Madrid, Spain \\ b Departamento de Física Teórica I, Facultad de Ciencias Físicas, Universidad Complutense de Madrid, 28040 Madrid, Spain \\ ${ }^{c}$ NIKHEF-H, Postbus 41882, 1009 DB Amsterdam, The Netherlands
}

Received 5 June 1995

Editor: P.V. Landshoff

\begin{abstract}
We regularize QCD using the combination of higher covariant derivatives and Pauli-Villars determinants proposed by Slavnov. It is known that for pure Yang-Mills theory the Pauli-Villars determinants generate unphysical logarithmic radiative corrections at one loop that modify the beta function. Here we prove that when the gauge fields are coupled to fermions so that one has $Q C D$, these unphysical corrections translate into a violation of unitarity. We provide an understanding of this by showing that Slavnov's choice for the Pauli-Villars determinants introduces extra propagating degrees of freedom that are responsible for the unitarity breaking. This shows that Slavnov's regularization violates unitarity, hence that it should be rejected.
\end{abstract}

\section{Introduction}

The advantages of using gauge invariant regularization methods are well known to the quantum field theory practitioner. The problem is that there are not so many gauge invariant regularization methods available. The two most popular ones proposed to date are probably dimensional regularization [1] and the method of higher covariant derivatives [2]. Dimensional regularization works well for vector gauge theories, for which the algebraic structure is not altered by a change in the number of dimensions of spacetime. Unfortunately, when it comes to chiral gauge theories, it is not clear [3,4] whether it is possible to consistently define dimensional regularization, the reason being that the properties of chiral objects depend on the dimensionality of spacetime and this conflicts somehow with the ideas behind dimensional regularization.
The situation for the method of higher covariant derivatives is more confusing. To describe it, we will restrict ourselves to QCD, the theory we will be discussing here. As is well known, higher covariant derivatives only provide a partial regularization, since they leave one-loop divergences unregularized. To achieve full regularization, a second regulator taking care of the unregularized one-loop divergences must be introduced. Choosing such a regulator is not a simple issue, since one would like to pick one that preserves gauge invariance and that at the same time does not jeopardize what has been gained at two and higher loops with the introduction of higher covariant derivatives. Slavnov [5] proposed in the seventies to use as second regulator a certain combination of gauge invariant Pauli-Villars determinants [see Eq. (8) for their expression]. Adopting his proposal, one ends up with a hybrid regularization that combines higher covariant derivatives with Pauli-Villars determinants 
of a certain form and that keeps the dimension of spacetime at its physical value. We will call this regularization prescription Slavnov's regularization and denote it by SR. Keeping the dimensionality of spacetime unchanged and preserving gauge invariance, SR looks like a good starting point to formulate a suitable regularization method for chiral gauge theories. Unfortunately, there is no agreement as for whether or not $\mathrm{SR}$ is a consistent regularization method. On the one hand, there are claims that (i) the Pauli-Villars determinants spoil regularization at two and higher loops [6] and (ii) that, even at one loop, renormalization is inconsistent with gauge invariance [7]. On the other, there are calls [8] rebating these claims.

To settle the dispute, and motivated by its potentiality for chiral gauge theories, SR was used in Ref. [9] to explicitly regularize and renormalize Yang-Mills theory at one loop. There it was proved that the PauliVillars determinants on which SR is based generate unphysical logarithmic radiative corrections that modify the beta function of the theory at one loop, giving for the latter an unphysical value. The purpose of this paper is to show that these unphysical corrections produce a violation of unitarity when the Yang-Mills fields are coupled to fermionic matter, so that one has full QCD.

\section{Slavnov's regularization}

Let us start by briefly recalling the basics of SR. We are interested in $N_{\mathrm{c}}$-coloured, $N_{\mathrm{f}}$-flavoured QCD in four dimensions. Not to fall short of rigour in the computation of Feynman integrals, we will work in Euclidean space and recover Minkowski spacetime results by Wick rotating the final results. In Euclidean space, QCD's classical action in a covariant gauge $\partial A^{a}+\alpha b^{a}=0$ takes the form

$S=\int d^{4} x\left(\mathcal{L}_{\mathrm{QCD}}+\mathcal{L}_{\mathrm{GF}}\right)$,

where $\mathcal{L}_{\mathrm{QCD}}$ and $\mathcal{L}_{\mathrm{GF}}$ are given by

$$
\begin{aligned}
& \mathcal{L}_{\mathrm{QCD}}=\frac{1}{4} F_{\mu \nu}^{a} F^{a \mu \nu}-\sum_{q=1}^{N_{\mathrm{f}}} \bar{\psi}_{q}\left(i \not \partial+i g A^{a} T^{a}-m_{q}\right) \psi_{q} \\
& \mathcal{L}_{\mathrm{GF}}=-\frac{\alpha}{2} b^{a} b^{a}-b^{a}\left(\partial A^{a}\right)+\bar{c}^{a}(\partial D c)^{a}
\end{aligned}
$$

and the notation is as follows. $A_{\mu}^{a}$ denotes the gauge field, $\bar{\psi}_{q}$ and $\psi_{q}$ the quark fields, $\bar{c}^{a}$ and $c^{a}$ the FaddeevPopov ghosts, $F_{\mu \nu}^{a}=\partial_{\mu} A_{\nu}^{a}-\partial_{\nu} A_{\mu}^{a}+g \int^{a b c} A_{\mu}^{b} A_{\nu}^{c}$ the field strength and $D_{\mu}^{a c}=\delta^{a c}+g f^{a b c} A_{\mu}^{b}$ the covariant derivative. $g$ is the coupling constant, $f^{a b c}$ are the structure constants of the gauge algebra, $T^{a}$ are the generators of the gauge algebra in the fundamental representation, $m_{q}$ are the fermion masses and $\alpha$ is the gauge-fixing parameter. The constants $f^{a b c}$ and the generators $T^{a}$ are normalized so that $f^{a c d} f^{b c d}=N_{\mathrm{c}} \delta^{a b}$ and $\operatorname{tr}\left(T^{a} T^{b}\right)=\frac{1}{2} \delta^{a b}$. SR regularizes QCD in two steps. First, it introduces a higher covariant derivative term and modifies the gauge-fixing part of the action so that now one has

$S_{\Lambda}=\int d^{4} x\left(\mathcal{L}_{\mathrm{QCD}}+\mathcal{L}_{\mathrm{HCD}}+\mathcal{L}_{\mathrm{G \Gamma}}^{\prime}\right)$

with

$\mathcal{L}_{\mathrm{HCD}}=\frac{1}{4 \Lambda^{4}}\left(D^{2} F_{\mu \nu}\right)^{a}\left(D^{2} F^{\mu \nu}\right)^{a}$

and

$\mathcal{L}_{\mathrm{GF}}^{\prime}=-\frac{\alpha}{2} b^{a} \frac{1}{f^{2}\left(\partial^{2} / \Lambda^{2}\right)} b^{a}-b^{a}\left(\partial A^{a}\right)+\bar{c}^{a}(\partial D c)^{a}$.

Here $\Lambda$ is a mass and $f\left(\partial^{2} / \Lambda^{2}\right)$ is a function that in momentum space is given by

$f=1+\frac{p^{4}}{\Lambda^{4}}$.

Some simple power counting shows that the only superficially divergent 1 PI Feynman diagrams generated by $S_{\Lambda}$ are the one-loop diagrams contributing to the two-, three- and four-point 1PI Green functions of the gauge field. Thus the modification of QCD's action along the lines of Eqs. (4)-(6) does not regularize the theory completely, but leaves some one-loop divergences unregularized. Before explaining Slavnov's idea to regularize the latter divergences, let us mention that the choice of $f\left(\partial^{2} / \Lambda^{2}\right)$ is somewhat arbitrary. Here we have chosen it as in (7) so as to ensure locality and make all $\alpha$-dependent contributions finite by power counting. Taking, e.g., $f=1+\left(p^{2} / \Lambda^{2}\right)$ also ensures locality but leaves $\alpha$-dependent contributions unregularized at one loop (see Ref. [9] for a discussion of this point). 
The second step in SR is to regularize the one-loop divergences generated by $S_{\Lambda}$. Slavnov [5] proposed to do this by introducing Pauli-Villars determinants in the generating functional so that it reads

$$
\begin{aligned}
Z & {[J, \chi, \zeta, \bar{\zeta}] } \\
& =\int \mathcal{D} A \mathcal{D} b \mathcal{D} \bar{\psi} \mathcal{D} \psi \mathcal{D} \bar{c} \mathcal{D} c e^{-\left(S_{\Lambda}+S_{\text {solure }}\right)} \\
& \times \prod_{i=1}^{I}\left(\operatorname{det} \mathbb{A}_{i}\right)^{-\alpha_{i} / 2}\left(\operatorname{det} \mathbb{C}_{i}\right)^{\alpha_{i}} \prod_{q=1}^{N_{\mathrm{f}}} \prod_{j=1}^{J_{q}}\left(\operatorname{det} \mathbb{F}_{q j}\right)^{\eta_{q j}}
\end{aligned}
$$

Here

$$
\begin{aligned}
& S_{\text {source }} \\
& \quad=\int d^{4} x\left[J^{a \mu} A_{\mu}^{a}+b^{a} \chi^{a}+\sum_{q=1}^{N_{\mathrm{f}}}\left(\bar{\psi}_{q} \zeta_{q}+\bar{\zeta}_{q} \psi_{q}\right)\right]
\end{aligned}
$$

is the source term coupling the fields $A_{\mu}^{a}, b^{a}, \psi_{q}$ and $\bar{\psi}_{q}$ to their external sources $J^{a \mu}, \chi^{a}, \bar{\zeta}_{q}$ and $\zeta_{q}$, and the determinants $\operatorname{det} \mathbb{A}_{i}$, det $\mathbb{C}_{i}$ and det $\mathbb{F}_{q j}$ are defined by

$$
\begin{aligned}
& \left(\operatorname{det} \mathbb{A}_{i}\right)^{-1 / 2}=\int \mathcal{D} A_{j} \delta\left(D A_{j}\right) \exp \left\{-\frac{1}{2} \int d^{4} x\right. \\
& \left.\times \int d^{4} y A_{j}^{a \mu}(x) O_{j \mu \nu}^{a b}(x-y) A_{j}^{b \mu}(y)\right\} \\
& \operatorname{det} \mathbb{C}_{i}=\operatorname{det}\left(-D^{2}+M_{i}^{2}\right) \\
& \operatorname{det} \mathbb{F}_{q j}=\operatorname{det}\left(i \not \partial+g A^{a} T^{a}+\mu_{q j}\right) .
\end{aligned}
$$

The parameters $\alpha_{i}$ and $\eta_{q j}$ are arbitrary real parameters satisfying the conditions

$$
\begin{aligned}
& \sum_{i=1}^{I} \alpha_{i}+1=0 \\
& \sum_{j=1}^{J_{q}} \eta_{q j}+1=0, \quad m_{q}^{2}+\sum_{j=1}^{J_{q}} \eta_{q j} \mu_{q j}^{2}=0,
\end{aligned}
$$

$M_{i}$ and $\mu_{q j}$ are masses and the operator $O_{j \mu \nu}^{a b}(x-y)$ in Eq. (9) is given by

$$
\begin{gathered}
o_{j \mu \nu}^{a b}(x-y)=\frac{\delta^{2} S_{\Lambda}}{\delta A_{\mu}^{a}(x) \delta A_{\nu}^{b}(y)} \\
+M_{j}^{2} \delta^{a b} g^{\mu \nu} \delta(x-y) .
\end{gathered}
$$

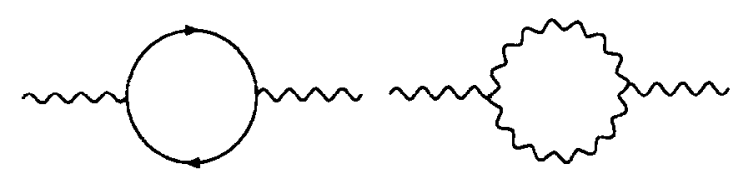

(a)

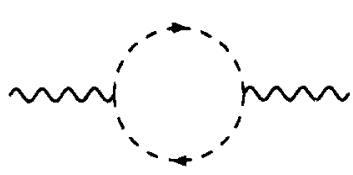

(c) (b)

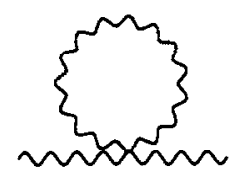

(d)
Fig. 1. Contributions from $S_{\Lambda}$ to the vacuum polarization tensor

Strictly speaking, Slavnov only considered pure YangMills theory, so he did not need to introduce determinants det $\mathbb{F}_{q j}$ to regularize the divergences generated by quarks running along internal loops. We will see, anyway, that the determinants det $\mathbb{F}_{q j}$ do not pose any problem and that the difficulties arise from the determinants $\operatorname{det} \mathbb{A}_{i}$. It is very easy to see [8] that $\operatorname{det} \mathbb{A}_{i}$, $\operatorname{det} \mathbb{C}_{i}$ and $\operatorname{det} \mathbb{F}_{q j}$ are gauge invariant. This, together with the gauge invariance of $S_{\Lambda}$, implies that the functional $Z[J, \chi, \zeta, \bar{\zeta}]$ satisfies the same BRS identities as the unregularized functional one would construct starting from QCD's action $S$ in Eq. (1). It can also be shown [5,9] that conditions (12) and (13) ensure that $Z[J, \chi, \zeta, \bar{\zeta}]$ generates finite Green functions at one loop. So, all in all, one has a generating functional which is manifestly gauge invariant and that generates finite Green functions at one loop.

It is very important to understand the regularization mechanism of one-loop divergences in $Z[J, \chi, \zeta, \bar{\zeta}]$. To this end, let us consider the vacuum polarization tensor it generates. From the action $S_{\Lambda}$, it receives the contributions of the diagrams in Fig. 1. In addition there are the contributions from $\prod_{i}\left(\operatorname{det} \mathbb{A}_{i}\right)^{-\alpha_{i} / 2}\left(\operatorname{det} \mathbb{C}_{i}\right)^{\alpha_{i}} \prod_{q} \prod_{j}\left(\operatorname{det} \mathbb{F}_{q j}\right)^{\eta_{q j}}$ in the measure of the path integral. Using the very same techniques as for ordinary Pauli-Villars regularization of QED, it is very easy to see that conditions (13) imply that the product $\prod_{q} \prod_{j}\left(\operatorname{det} \mathbb{F}_{q j}\right)^{\eta_{q j}}$ regularizes the divergences in diagram 1a. As for diagrams $1 \mathrm{~b}-$ 1d, Slavnov [5] has argued using formal path integral manipulations that violate locality that the product $\prod_{i}\left(\operatorname{det} \mathbb{A}_{i}\right)^{-\alpha_{i} / 2}\left(\operatorname{det} \mathbb{C}_{i}\right)^{\alpha_{i}}$ cancels the divergences in diagrams $1 \mathrm{~b}-1 \mathrm{~d}$ provided Eq. (12) and the extra condition 
$\sum_{i=1}^{\prime} \alpha_{i} M_{i}^{2}=0$

are met. It has been shown [9] that, to check whether this is actually the case within the framework of local regularization, an extra regulator $\mathcal{R}$ must be introduced. It turns out that after introducing such a regulator and performing calculations at finite $\mathcal{R}$, the 2-point divergences that arise in $\prod_{i}\left(\operatorname{det} \mathbb{A}_{i}\right)^{-\alpha_{i} / 2}\left(\operatorname{det} \mathbb{C}_{i}\right)^{\alpha_{i}}$ when $\mathcal{R} \rightarrow 0$ cancel the divergences that arise in diagrams $1 \mathrm{~b}-1 \mathrm{~d}$ when $\mathcal{R} \rightarrow 0$, provided only condition (12) is satisfied. So indeed the sum of diagrams 1a-1d with the 2-point corrections from $\prod_{i}\left(\operatorname{det} \mathbb{A}_{i}\right)^{-\alpha_{i} / 2}\left(\operatorname{det} \mathbb{C}_{i}\right)^{\alpha_{i}} \prod_{q} \Pi_{j}\left(\operatorname{det} \mathbb{F}_{q j}\right)^{\eta_{q j}}$ is finite if Eqs. (12) and (13) hold, but to see it without giving up locality, an extra regulator $\mathcal{R}$ is needed. The fact that one has to introduce an extra regulator $\mathcal{R}$ means strictly speaking that by itself SR does not provide a local regularization of QCD.

Having a generating functional that generates finite Green functions for finite values of the masses $\Lambda, M_{i}$ and $\mu_{q j}$ is not all. One has to devise a subtraction procedure that removes the divergences associated to large values of the regulators $\Lambda, M_{i}$ and $\mu_{q j}$, while preserving gauge invariance. There have been claims in the past [7] that such a procedure does not exist for pure Yang-Mills theory and that this is enough to kill SR. In Ref. [9], however, it has been proved that this is not the case and the most general subtraction procedure consistent with gauge invariance for purc Yang-Mills theory has been given. Its generalization to QCD being straightforward, we will not present here. In what follows, we show that the functional $Z[J, \chi, \zeta, \bar{\zeta}]$ generates unphysical contributions that, after Wick rotation to Minkowski spacetime, spoil unitarity.

\section{Violation of unitarity}

Let us now come to Minkowski spacetime, the correct framework to discuss unitarity. As is well known, unitarity implies that the transition amplitude $T_{f i}$ for a physical process $|i\rangle \rightarrow|f\rangle$ must satisfy the relation

$2 \operatorname{Im} T_{f i}=\sum_{n}(2 \pi)^{4} \delta^{4}\left(p_{n}-p_{i}\right) T_{n f}^{*} T_{n i}$,

where the sum is extended over all physical intermediate states $|n\rangle$ connecting $|i\rangle$ with $|f\rangle$ and $p_{n}$ denotes the momentum of the state $|n\rangle$. Consider the process fermion, antifermion going to fermion, antifermion. For this process, Eq. (14) takes at first order in perturbation theory the form

$$
2 \operatorname{Im} T_{1, \overline{\mathrm{f}} \rightarrow \overline{\mathrm{ff}}}=\sum_{n}(2 \pi)^{4} \delta^{4}\left(p_{n}-p_{i}\right)\left|T_{0, n \rightarrow \overline{\mathrm{ff}}}\right|^{2} .
$$

The renormalized amplitude $T_{1} \equiv T_{\mathrm{I}, \overline{\mathrm{ff}} \rightarrow \overline{\mathrm{ff}}}$ can be computed by first regularizing and then by subtracting the divergences associated to the particular regulator one has used. This way, the left-hand side becomes regularization and subtraction-dependent. Actually, regularization-dependent only, since different admissible subtraction schemes differ by finite local renormalizations and these carry finite local radiative corrections which do not reach the imaginary part of the transition amplitude. The right-hand side, however, is regularization and subtraction-independent, since it only involves the Feynman rules of the unregularized theory. Hence Eq. (15) can be viewed as a necessary condition that the particular regularization and subtraction prescriptions used to renormalize the theory must satisfy in order to preserve unitarity. The idea of our proof of violation of unitarity by SR is to compute $\operatorname{Im} T_{1}$ in any SR-based renormalization scheme and see that it does not satisfy Eq. (15). Now, since dimensional regularization (DR) preserves unitarity, the right-hand side in Eq. (15) is equal to twice the imaginary part of $T_{1}$ as computed in any DR-based renormalization scheme. Thus Eq. (15) can be replaced with

$2 \operatorname{Im} T_{1, \mathrm{SR}}=2 \operatorname{Im} T_{1, \mathrm{DR}}$,

where the subscripts SR and DR refer to the regularization method used to compute $T_{1}$. In the following we show that Eq. (16) does not hold.

As is well known, the imaginary part of the amplitude $T_{1}$ receives contributions from the diagrams depicted in Fig. 2, where all external legs are on-shell and the shadowed bubble stands for the vacuum polarization tensor at one loop. To compute the contribution of these diagrams to $\operatorname{Im} T_{1, \mathrm{SR}}$, we proceed as follows. We first calculate the renormalized contribution of every diagram to the amplitude $T_{1, \mathrm{SR}}$ in Euclidean space; once we have done this, we Wick rotate to Minkowski spacetime; finally, we take the imaginary 


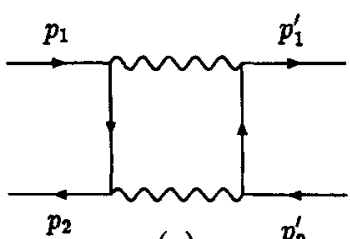

(a)

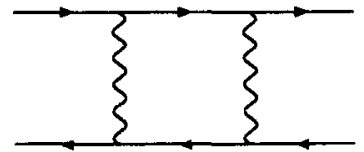

(b)

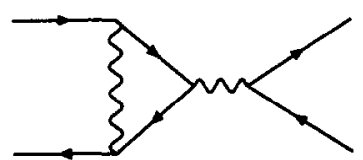

(c)

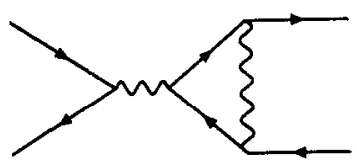

(d)

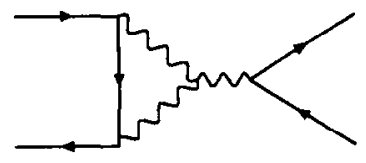

(e)

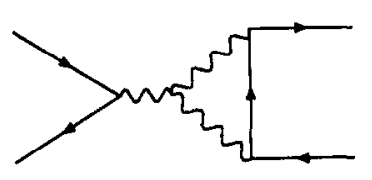

(f)

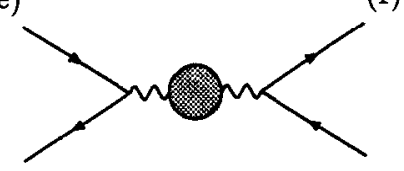

(g)

Fig. 2. Diagrams that contribute to the imaginary part of $T_{1, \overline{\mathrm{ff}} \rightarrow \overline{\mathrm{ff}}}$

part. As concerns the technical aspects of this computation, we note that the calculation of the renormalized contribution of any of the diagrams involved requires computing its limit $\Lambda, M_{i}, \mu_{q j} \rightarrow \infty$ and subtracting the divergences associated to this limit. The evaluation of such limit is tedious but straightforward if one uses the techniques developed in Refs. [4] and [9]. For simplicity, and since transition amplitudes are independent of the gauge fixing parameter $\alpha$, we will work in the Feynman gauge $\alpha=1$.

Wc start by looking at diagrams $2 a$ and $2 b$. If we amputate the external legs, we are left in both instances with a 1PI diagram whose expression in Euclidean space in the Feynman gauge is

$G_{4}\left(p_{1}, p_{2}, p_{1}^{\prime} ; \Lambda\right)=g^{4} T^{a} T^{b} T^{b} T^{a} \sum_{q=1}^{N_{f}} I_{q}(\Lambda)$,

where

$I_{q}(\Lambda)=\int \frac{d^{4} k}{(2 \pi)^{4}}$

$$
\begin{aligned}
& \times \frac{\gamma_{\mu}\left(\not p_{1}+\not k-m_{q}\right) \gamma_{\nu} \gamma_{\rho}\left(\not p_{1}^{\prime}+\not k-m_{q}\right) \gamma_{\lambda}}{\left[\left(p_{1}+k\right)^{2}+m_{q}^{2}\right]\left[\left(p_{1}^{\prime}+k\right)^{2}+m_{q}^{2}\right]} \\
& \times D^{\mu \lambda}\left(p_{1}-p_{2}+k, \Lambda\right) D^{\nu \rho}(k, \Lambda)
\end{aligned}
$$

and

$$
D_{\mu \nu}(k, \Lambda)=\frac{\Lambda^{4}}{k^{4}+\Lambda^{4}}\left(\frac{g_{\mu \nu}}{k^{2}}-\frac{k_{\mu} k_{\nu}}{k^{4}+\Lambda^{4}}\right)
$$

is the gluon propagator for the action $S_{\Lambda}$ in Eq. (4) with $\alpha=1$. Using the Lebesgue dominated convergence theorem, it is straightforward to see that the $\Lambda \rightarrow \infty$ limit of $I_{q}(\Lambda)$ is well defined and equal to

$$
\begin{aligned}
& \lim _{\Lambda \rightarrow \infty} I_{q}(\Lambda)=4 \int \frac{d^{4} k}{(2 \pi)^{4}} \\
& \quad \times \frac{\gamma_{\mu}\left(\not_{1}+k-m_{q}\right)\left(\not p_{1}^{\prime}+k-m_{q}\right) \gamma^{\mu}}{\left[\left(p_{1}+k\right)^{2}+m_{q}^{2}\right]\left[\left(p_{1}^{\prime}+k\right)^{2}+m_{q}^{2}\right]\left(p_{1}-p_{2}+k\right)^{2} k^{2}} .
\end{aligned}
$$

Hence no infinite renormalization is necessary. This is no surprise since the unregularized 1PI four-fermion vertex $G_{4}$ at one loop is finite by power counting and is given by the right hand-side in Eq. (17). This implies that the renormalized four-vertex $G_{4}$ at one loop as computed with SR agrees with the renormalized four-vertex $G_{4}$ as computed with any other acceptable regularization method, and in particular with DR. It follows then, after Wick rotating to Minkowski space and putting the external legs on-shell, that the contribution of diagrams $2 a$ and $2 b$ to the imaginary part of $T_{1}$ in any SR-based renormalization scheme is the same as in any DR-based renormalization scheme:

$\operatorname{Im} T_{1, \mathrm{SR}}^{2 a, 2 b}=\operatorname{Im} T_{1, \mathrm{DR}}^{2 a, 2 b}$

Next we move on to diagrams $2 \mathrm{c}$ and $2 \mathrm{~d}$. They both have the 1PI diagram in Fig. 3 as subdiagram. For the latter subdiagram, SR gives in Euclidean space and in the Feynman gauge

$$
\begin{aligned}
\Gamma_{\mu}^{a}\left(p_{1}, p_{2}, \Lambda\right)=i g^{3} \frac{T^{a}}{2 N_{c}} \sum_{q=1}^{N_{f}} \int \frac{d^{4} k}{(2 \pi)^{4}} \\
\quad \times \frac{\gamma_{\lambda}\left(p_{2}+k-m_{q}\right) \gamma_{\mu}\left(\not p_{1}+k-m_{q}\right) \gamma_{\nu}}{\left[\left(p_{2}+k\right)^{2}+m_{q}^{2}\right]\left[\left(p_{1}+k\right)^{2}+m_{q}^{2}\right]} \\
\quad \times D^{\lambda \nu}(k, \Lambda) .
\end{aligned}
$$




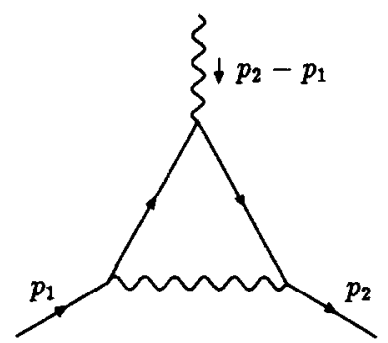

Fig. 3. IPI subdiagrams in Figs. $2 \mathrm{c}$ and $2 \mathrm{~d}$.

Using the techniques in Ref. [4] to compute the large$\Lambda$ limit, and the results in Ref. [10] to rewrite the contributions that do not vanish in this limit, we obtain

$$
\begin{aligned}
& \lim _{\Lambda \rightarrow \infty} \Gamma_{\mu}^{a}\left(p_{1}, p_{2}, \Lambda\right)=\frac{i g^{3}}{8 \pi^{2}} \frac{T^{a}}{2 N_{c}} \\
& \times \sum_{q=1}^{N_{\mathrm{f}}}\left\{\gamma_{\mu}\left[\frac{1}{2} \ln \left(\frac{\Lambda^{2}}{m_{q}^{2}}\right)+v_{0}\right]\right. \\
& \left.\quad+F_{\mu}^{\mathrm{fin}}\left(m_{q}, p_{1}, p_{2}\right)\right\} .
\end{aligned}
$$

where $v_{0}$ is a numerical constant and the finite part is given by

$F_{\mu}^{\mathrm{fin}}\left(m_{q}, p_{1}, p_{2}\right)=\int_{0}^{1} d x \int_{0}^{1} d y y \ln \left[\frac{m_{q}^{2}}{D(x, y)}\right]$

$+\int_{0}^{1} d x \int_{0}^{1} d y$

$\times \frac{m_{q}^{2} \gamma_{\mu}-2 m_{q}\left(p_{1}+p_{2}-2 y \bar{p}\right)_{\mu}+\left(\not p_{1}-y \bar{p}\right) \gamma_{\mu}\left(p_{2}-y \bar{p}\right)}{D(x, y)}$

Here $D(x, y)$ and $\bar{p}^{\mu}$ stand for

$D(x, y)=m_{q}^{2}+\left(p_{2}-p_{1}\right)^{2} x(1-x)+y(1-y) \bar{p}^{2}$

and

$\bar{p}^{\mu}=(1-x) p_{1}^{\mu}+x p_{2}^{\mu}$.

We see that $\Gamma_{\mu}^{a}\left(p_{1}, p_{2}, \Lambda\right)$ diverges as $\Lambda \rightarrow \infty$. To remove the divergence, we perform the most general subtraction compatible with gauge invariance and obtain

$$
\Gamma_{\mu, \operatorname{ren}}^{a}\left(p_{1}, p_{2}, \sigma\right)=\frac{i g^{3}}{8 \pi^{2}} \frac{T^{a}}{2 N_{c}}
$$

$$
\begin{aligned}
& \times \sum_{q=1}^{N_{\mathrm{t}}}\left\{\frac{1}{2} \gamma_{\mu}\left[-\ln \left(\frac{m_{q}^{2}}{\sigma^{2}}\right)+v\left(\frac{m_{q}^{2}}{\sigma^{2}}\right)\right]\right. \\
& \left.+F_{\mu}^{\mathrm{fin}}\left(m_{q}, p_{1}, p_{2}\right)\right\},
\end{aligned}
$$

$\sigma$ being the renormalization mass scale and $v\left(m_{q}^{2} / \sigma^{2}\right)$ a finite function that does not depend on the momenta and that is only restricted by BRS invariance (in a minimal scheme, it would be zero). Let us now recall what DR gives. In DR, instead of Eq. (19) one has

$$
\begin{aligned}
& \lim _{\varepsilon \rightarrow 0} \Gamma_{\mu}^{a}\left(p_{1}, p_{2} ; \varepsilon, \nu\right)=\frac{i g^{3}}{8 \pi^{2}} \frac{T^{a}}{2 N_{c}} \\
& \quad \times \sum_{q=1}^{N_{f}}\left\{\frac{1}{2} \gamma_{\mu}\left[\frac{1}{\varepsilon}+\ln \left(\frac{\nu^{2}}{m_{q}^{2}}\right)+v_{0}\right]\right. \\
& \left.\quad+F_{\mu}^{\text {fin }}\left(m_{q}, p_{1}, p_{2}\right)\right\},
\end{aligned}
$$

where $\nu$ is the dimensional regularization mass scale and $v_{0}$ is a constant different from that in Eq. (19). After renormalization, one obtains the same expression as in Eq. (21), modulo finite local radiative corrections. This implies, after Wick rotating to Minkowski space and replacing the subdiagram in Fig. 3 with its renormalized expression, that the imaginary part of diagrams $2 \mathrm{c}$ and $2 \mathrm{~d}$ is the same for SR-based renormalization schemes as for DR-based renormalization schemes:

$\operatorname{Im} T_{1, \mathrm{SR}}^{2 c, 2 d}=\operatorname{Im} T_{1, \mathrm{DR}}^{2 c, 2 d}$.

Proceeding analogously, it is easy to see that the same holds true for diagrams $2 \mathrm{e}$ and $2 \mathrm{f}$ :

$\operatorname{Im} T_{1, \mathrm{SR}}^{2 e, 2 f}=\operatorname{Im} T_{1, \mathrm{DR}}^{2 e, 2 f}$.

Let us finally look at diagram $2 \mathrm{~g}$, and let us concentrate on the one-loop vacuum polarization tensor $\Pi_{\mu \nu}^{a b}(k)$ hidden in it. The latter is made of two contributions. First there is the contribution (we will denote it with a prime) from diagram $1 \mathrm{~d}$ and the determinants det $\mathbb{F}_{q j}$ that regularize it. Its expression can be computed following the very same steps as for the vacuum polarization tensor in Pauli-Villars regularized QED [11]. After some calculations, we get

$\Pi_{\mu \nu}^{\prime a b}\left(k, \mu_{q j}\right)=-\frac{g^{2}}{16 \pi^{2}} \delta^{a b}$ 


$$
\begin{aligned}
& \times \frac{2}{3} \sum_{q=1}^{N_{1}}\left[\sum_{j=1}^{J_{q}} \eta_{q j} \ln \left(\frac{m_{q}^{2}}{\mu_{q j}^{2}}\right)+h\left(\frac{m_{q}^{2}}{k^{2}}\right)+\pi_{0}^{\prime}\right] \\
& \times\left(k_{\mu} k_{\nu}-k^{2} g_{\mu \nu}\right),
\end{aligned}
$$

where $h(x)$ is the function

$h(x)=4 x+(1-2 x) \sqrt{1+4 x} \ln \left(\frac{\sqrt{1+4 x}+1}{\sqrt{1+4 x}-1}\right)$

and $\pi_{0}^{\prime}$ is a numerical constant. Then there is the contribution from diagrams $1 \mathrm{~b}-1 \mathrm{~d}$ and from the PauliVillars determinants $\operatorname{det} \mathbb{A}_{i}$ and $\operatorname{det} \mathbb{C}_{i}$. This contribution (we will denote it with a double prime) is the same as for pure Yang-Mills theory, and its $\Lambda, M_{i} \rightarrow$ $\infty$ limit has been computed for arbitrary $\alpha$ in Ref. [9]. Borrowing the results from there, we have that in the Feynman gauge

$$
\begin{aligned}
& \Pi_{\mu \nu}^{\prime \prime a b}\left(k, \Lambda, M_{i}\right)=\frac{g^{2} N_{\mathrm{c}}}{16 \pi^{2}} \delta^{a b} \\
& \quad \times\left[A_{2} \ln \left(\frac{k^{2}}{\Lambda^{2}}\right)+B_{2} \sum_{i=1}^{I} \alpha_{i} \ln \left(\frac{k^{2}}{M_{i}^{2}}\right)+\pi_{0}^{\prime \prime}\right] \\
& \quad \times\left(k_{\mu} k_{\nu}-k^{2} g_{\mu \nu}\right)
\end{aligned}
$$

where

$$
A_{2}-B_{2}=\frac{11}{6}
$$

and $\pi_{0}^{\prime \prime}$ is another numerical constant. The actual values of $A_{2}$ and $B_{2}$ depend on the way the masses $\Lambda$ and $M_{i}$ are sent to infinity. For example, sending $\Lambda$ to infinity while keeping $M_{i}$ finite, and taking in the result $M_{i} \rightarrow \infty$ gives different $A_{2}$ and $B_{2}$ as proceeding the other way around. The difference $A_{2}-B_{2}$ is, however, independent of the path followed to approach $\Lambda, M_{i} \rightarrow \infty$ and is always given by Eq. (26). Summing the contributions (24) and (25), performing the most general subtraction compatible with gauge invariance, and Wick rotating to Minkowski space, we obtain for the renormalized vacuum polarization tensor

$$
\begin{aligned}
& \Pi_{\mu \nu, \text { ren }}^{a b}(k, \sigma) \\
& \quad=\frac{g^{2}}{16 \pi^{2}} \Pi_{\mathrm{SR}}\left(k^{2}, \sigma\right) \delta^{a b}\left(k_{\mu} k_{\nu}-k^{2} g_{\mu \nu}\right),
\end{aligned}
$$

where

$$
\begin{aligned}
\Pi_{\mathrm{SR}}\left(k^{2}, \sigma\right) & =\frac{11 N_{\mathrm{c}}}{6} \ln \left(-\frac{k^{2}}{\sigma^{2}}\right) \\
- & \frac{2}{3} \sum_{q=1}^{N_{\mathrm{f}}}\left[h\left(-\frac{m_{q}^{2}}{k^{2}}\right)+\ln \left(\frac{m_{q}^{2}}{\sigma^{2}}\right)+\pi\left(\frac{m_{q}^{2}}{\sigma^{2}}\right)\right],
\end{aligned}
$$

$\sigma$ is the subtraction point and $\pi\left(m_{q}^{2} / \sigma^{2}\right)$ is an arbitrary real function carrying local finite radiative corrections constrained only by BRS invariance. For momenta $k^{\mu}$ such that $k^{2}>0$, the vacuum polarization tensor picks an imaginary part since

$$
\begin{aligned}
& \operatorname{Im} \Pi_{\mathrm{SR}}\left(k^{2}, \sigma\right)=\frac{11 N_{\mathrm{c}}}{6} \theta\left(k^{2}\right) \\
& \quad+\frac{2}{3} \sum_{q=1}^{N_{\mathrm{f}}} \theta\left(k^{2}-4 m_{q}^{2}\right)\left(1+\frac{2 m_{q}^{2}}{k^{2}}\right) \sqrt{1-\frac{4 m_{q}^{2}}{k^{2}}} .
\end{aligned}
$$

Let us compare this with the DR result. We recall that in any DR-based subtraction scheme, one has in the Feynman gauge that

$$
\begin{aligned}
& \operatorname{Im} \Pi_{\mathrm{DR}}\left(k^{2}, \sigma\right)=\frac{5 N_{\mathrm{c}}}{3} \theta\left(k^{2}\right) \\
& \quad+\frac{2}{3} \sum_{q=1}^{N_{\mathrm{f}}} \theta\left(k^{2}-4 m_{q}^{2}\right)\left(1+\frac{2 m_{q}^{2}}{k^{2}}\right) \sqrt{1-\frac{4 m_{q}^{2}}{k^{2}}} .
\end{aligned}
$$

We see that the coefficient in front of $\theta\left(k^{2}\right)$ is different from that in Eq. (29). As explained in Ref. [9], the difference $\frac{11}{6}-\frac{5}{3}=\frac{1}{6}$ is originated by the Pauli-Villars determinants det $\mathbb{A}_{i}$. It is plain now that the imaginary part of the renormalized contribution

$$
\begin{aligned}
T_{1}^{2 g} & =g^{2}\left[\bar{v}\left(p_{2}\right) \gamma^{\mu} T^{a} u\left(p_{1}\right)\right] \Pi\left(k^{2}, \sigma\right) \\
& \times \frac{1}{k^{4}}\left(k_{\mu} k_{\rho}-k^{2} g_{\mu \rho}\right)\left[\bar{u}\left(p_{1}^{\prime}\right) \gamma^{\rho} T^{a} v\left(p_{1}^{\prime}\right)\right]
\end{aligned}
$$

of diagram $2 \mathrm{~g}$ to the amplitude $T_{1}$ is not the same for SR-based renormalization schemes as for DR-based schemes. In other words,

$\operatorname{Im} T_{1, \mathrm{SR}}^{2 g} \neq \operatorname{Im} T_{1, \mathrm{DR}}^{2 g}$.

Putting together Eqs. (18), (22), (23) and (31), we have that

$\operatorname{Im} T_{1, \mathrm{SR}} \neq \operatorname{Im} T_{1, \mathrm{DR}}$, 
as we wanted to prove.

\section{Conclusion}

At this point we can draw the following conclusions:

(i) The regularization method proposed by Slavnov violates unitarity, the violation being produced by the Pauli-Villars determinants $\operatorname{det} \mathbb{A}_{i}$ that the method chooses. Let us try to gain some intuition of why this is so. Assume that we naively switch off the regulators in the regularized path integral in Eq. (8), that is to say, that we send the masses $\Lambda, M_{i}$ and $\mu_{q j}$ to infinity. Then we should recover the unregularized QCD path integral. However, this is not the case $[9,12]$. To see the latter, we rescale [12] the Pauli-Villars field $A_{i \mu}^{a} \rightarrow M^{-1} A_{i \mu}^{a}$ in $\left(\operatorname{det} \mathbb{A}_{i}\right)^{-1 / 2}$, take the limit $\Lambda, M_{j} \rightarrow \infty$, exponentiate $\delta\left(D A_{i}\right)$ and integrate over $d^{4} x$ once by parts. This leaves us with

$$
\begin{aligned}
& \left(\operatorname{det} \mathbb{A}_{i}\right)^{-1 / 2} \sim \int \mathcal{D} A_{i} \mathcal{D} b_{i} \\
& \quad \times \exp \left\{-\frac{1}{2} \int d^{4} x\left(A_{i}^{2}+2 A_{i} D b_{i}\right)\right\}
\end{aligned}
$$$$
\text { as } \Lambda, M_{i} \rightarrow \infty \text {. }
$$

Completing the square in the exponent and performing the integral yields $\left(\operatorname{det} D^{2}\right)^{-1 / 2}$. Since each $\left(\operatorname{det} \mathbb{A}_{i}\right)^{-1 / 2}$ is exponentiated to the power $\alpha_{i}$ and the $\alpha_{i}$ 's satisfy Eq. (12), we obtain a factor $\left(\operatorname{det} D^{2}\right)^{1 / 2}$. As for the determinants $\operatorname{det} \mathbb{C}_{i}$ and $\operatorname{det} \mathbb{F}_{q j}$, it is straightforward to see that their limits $M_{i} \rightarrow \infty$ and $\mu_{q j} \rightarrow \infty$ give unity. Thus taking the naive $\Lambda, M_{i}, \mu_{q j} \rightarrow \infty$ limit in $Z[J, \chi, \zeta, \bar{\zeta}]$ yields the unregularized QCD path integral plus an extra $\left(\operatorname{det} D^{2}\right)^{1 / 2}$. This extra determinant introduces propagating degrees of freedom that couple to the gluon field through the covariant derivative and which are not present in QCD's action. In other words, SR modifies QCD even at the tree level. Obviously the properties of the modified QCD are not the same as those of the true QCD. In the light of this, it is very easy to understand SR's violation of unitarity. What $T_{1, \mathrm{SR}}$ is really standing for is the transition amplitude $T_{1}$ for the modified theory. By the cutting rules of ' $t$ Hooft and Veltman [13], $\operatorname{Im} T_{1, S R}$ will receive contributions from the new propagating degrees of freedom. Hence there is no way $\operatorname{Im} T_{1, \mathrm{SR}}$ will agree with the imaginary part of $T_{1, \mathrm{QCD}} \equiv T_{1, \mathrm{DR}}$. All this discards SR as an acceptable regularization method.

(ii) Note that the diagrams whose rcgularization only involves $\Lambda$, namely diagrams $2 \mathrm{a}$ to $2 \mathrm{f}$, give the correct contribution to $T_{1, \mathrm{QCD}}$ in the $\Lambda \rightarrow \infty$ limit. This indicates that the higher covariant derivatives terms in Eq. (5) by themselves do not cause problems, in agreement with [14]. The question that remains open is to supplement higher covariant derivatives with a suitable local regularization that preserves gauge invariance manifestly. Let us recall in this regard that for a local regularization method to be such, it must provide integrals over loop momenta which are finite by power counting (this is what local regularization is about). If a prescription does not provide this finiteness by power counting, it should not be called a local regularization; not even in the event that the various divergent contributions from different divergent Feynman integrals cancel among themselves when the latter are properly defined through yet another regularization.

\section{References}

[1] G. 't Hooft and M. Veltman, Nucl Phys. B 44 (1972) 189; D.A. Akyeampong and R. Delbourgo, Nuov. Cim. A 17 (1973) 578; A 18 (1973) 94; A 19 (1974) 219.

[2] A.A. Slavnov, Nucl. Phys. B 31 (1971) 301; B.W. Lee and J. Zinn-Justin, Phys. Rev. D 5 (1972) 3121.

[3] C.P. Martin, Phys. Lett. B 241 (1990) 513.

[4] G. Giavarini, C.P. Martin and F. Ruiz Ruiz, Nucl. Phys. B 381 (1992) 222.

[5] A.A. Slavnov, Theor. Math. Phys. 33 (1977) 977.

[6] B.J. Warr, Ann. Phys. 183 (1988) 1.

[7] R. Sénéor, Some remarks for the construction of Yang-Mills theories, in: Renormalization of quantum field theories with non-linear field transformations, edited by $P$. Breitenlohner, D. Maison and K. Sibold (Springer-Verlag, Berlin 1988).

[8] L.D. Faddeev and A.A. Slavnov, Gauge fields, introduction to quantum theory (Benjamin 1991).

[9] C.P. Martin and F. Ruiz Ruiz, Nucl. Phys. B 436 (1995) 545.

[10] R. Kawabe, K.i. Aoki, Z. Hioki, M. Konuma and T. Muta, Prog. Theor. Phys. Suppl. 73 (1982) 1.

[11] C. Itzykson and J.B. Zuber, Quantum field theory (McGrawHill, 1987)

[12] M. Asorey and F. Falceto, On the consistency of regularization by higher covariant derivatives, University of Zaragoza preprint DFTUZ-95.3.

[13] G. 't Hooft and M. Veltman, Diagrammar, in: Particle interactions at very high energies, edited by $D$. Speiser, F. Halzen and J. Weyers (Plenum Press, London, 1974).

[14] C.P. Martin and F. Ruiz Ruiz, Phys. Lett. B 343 (1995) 218. 